\title{
Modeling day time turbulence profiles: Application to Teide Observatory
}

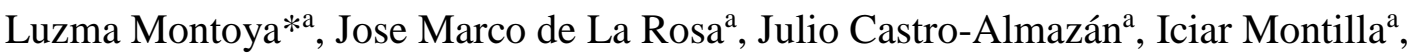 \\ Manuel Collados ${ }^{\mathrm{a}}$ \\ ${ }^{a}$ Instituto de Astrofísica de Canarias, Vía Lactea s/n 382005 La Laguna, Tenerife
}

\begin{abstract}
The new generation solar telescope, EST, will be equipped with MCAO capabilities. Site testing is critical for an optimal MCAO system design. The aim of this work is to estimate the refractive index structure constant profile, $\mathrm{Cn}^{2}$, at Observatorio del Teide (Canary Islands) using atmospheric data provided by radiosondes launched from sea level at Güimar station (15 km distant). There are different parametric models for deriving vertical turbulence profiles of $\mathrm{Cn}^{2}$ present in the literature. In this paper, several of those models are reviewed and the model that best fits is selected by correlating with the nighttime IAC-SCIDAR database.
\end{abstract}

Keywords: Turbulence Profiling, MCAO, Solar Adaptive optics

\section{INTRODUCTION}

The characterization of the atmospheric turbulence is critical for the design of an adaptive optics system. The refractive index structure constant, $\mathrm{Cn}^{2}$, is a measure of the turbulence strength [1] . This parameter depends on the site, altitude and time of day. In the boundary layer the $\mathrm{Cn}^{2}$ variation is dominated by heat exchange with the earth while in the free atmosphere it is dominated by wind shear and gravity waves.

The measure of $\mathrm{Cn}^{2}$ with altitude is not an easy task. $\mathrm{Cn}^{2}$ turbulence profiles can be derived from optical correlation methods using scintillometers (SCIDAR [2], SHABAR [3]) or by the application of turbulence models to atmospheric data provided by radiosondes.

At the Canary Islands observatories we are equipped with a SCIDAR instrument capable of deriving turbulence profiles during night time. A SHABAR instrument is also available to measure the ground layer profile during the day. However there is no instrument which provides direct $\mathrm{Cn}^{2}$ profiles for the free layer during day time.

The aim of this work is to derive a method to characterize the day time turbulence in the whole atmosphere.

The strategy to retrieve the $\mathrm{Cn}^{2}$ day time profiles is:

1. Derive turbulence profiles from night radiosondes data using different models.

2. Cross-check the models with SCIDAR data obtained during night time.

3. Choose the model that best fits to SCIDAR data.

4. Apply the model to radiosondes day-time data.

5. Combine ground layer SHABAR profiles with free atmosphere radiosonde profiles.

Non parametric models depend only on altitude and they do not take into account stratification. Parametric models include the dependence with site, stratification and meteorological parameters.

There is a large number of models. We have focused our study in four different parametric models which will be described in the following sections. 


\section{DESCRIPTION OF DATA SETS}

For this study we have compiled several sets of data.

We have an archive of SCIDAR data at Observatorio del Teide from 2003 to 2008 [1]. Those files provide information of the integrated $\mathrm{r}_{0}$ and vertical $\mathrm{Cn}^{2}$ profiles with a resolution of 300 meters. We have averaged the data from $00 \mathrm{UT}$ to $01 \mathrm{UT}$, which is the flight time of the radiosonde balloons.

We also have an archive with meteorological data from radiosondes at 12UT and 00UT. The balloon is launched from sea level at Güimar station. Two set of data are available: Low resolution data (LR), with samples taken every 10 seconds and a spatial resolution between 100-300 meters, downloaded from http://weather.uwyo.edu/upperair/sounding.html with the format shown in Table 1; and high resolution (HR) data, with samples acquired every 2 seconds and a spatial resolution of 10 meters, obtained from AEMET (Agencia Española de Meteorología) database (private communication)

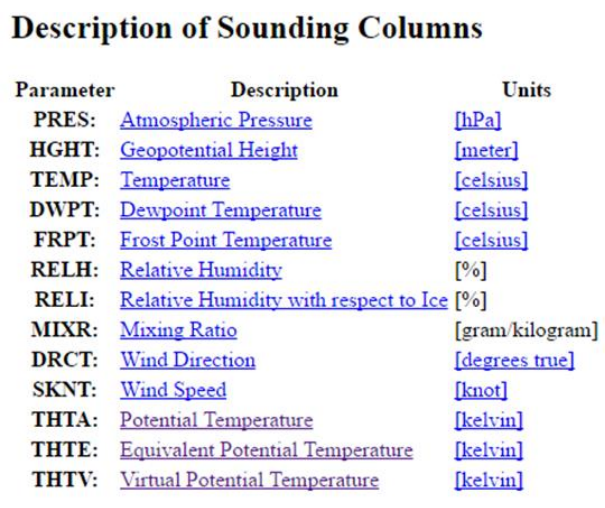

Table 1. Parameters measured by the radiosondes

Finally, we have an archive with SHABAR data obtained during the campaigns 2010 to 2014 [2]. Those data provide $\mathrm{Cn}^{2}$ profiles and integrated $\mathrm{r}_{0}$ values with a resolution of the order of meters. The main drawback of this instrument is that it is not sensitive to heights above $2 \mathrm{~km}$ due to an intrinsic limitation of the instrument.

\section{DESCRIPTION OF PARAMETRIC MODELS}

In the literature there exists a large number of models to retrieve the $\mathrm{Cn}^{2}$ function from meteo values. Most of them are suitable for the free atmosphere and are based on the relation obtained by Tatarski [1] which relates $\mathrm{Cn}^{2}$ with the gradient of refractive index, $\mathrm{M}$, and the outer scale, $\mathrm{L}_{0}$, by the following expression:

$$
C_{n}^{2}=\gamma M^{2} L_{0}^{\frac{4}{3}}
$$

where $\gamma$ is a constant that depends on the model and turbulence stability. In the following, we summarize how the models apply Tatarski's equation to compute $\mathrm{Cn}^{2}$.

\subsection{Coulman's Model}

Coulman et al. [4] proposed a model which fits the outer scale from measured values of the refractive index of structure, $\mathrm{Cn}^{2}$, and atmospheric variables. Coulman et al. derive the gradient of potential refractive index, $\mathrm{M}$, as: 


$$
M=-77.6 \times 10^{-6} \frac{P}{T}\left(\frac{\partial \ln \theta}{\partial z}\right) x\left[1+\frac{15500}{T}\left(q-0.5\left(\frac{\partial q / \partial z}{\partial \ln \theta / \partial z}\right)\right)\right]
$$

where $\mathrm{P}$ is the pressure in Hpa, $\mathrm{T}$ is the temperature in Kelvin, $\mathrm{q}$ is the humidity, $\theta$ is the potential temperature in Kelvin and $\mathrm{z}$ is the height in meters. In this model $\gamma$ is set to 2.8 which is a standard value used for the atmosphere derived by Tatarski [1].

$\mathrm{Cn}^{2}$ values are retrieved from SCIDAR data [2]. The $\mathrm{Cn} 2$ values have been averaged from 00UT to $01 \mathrm{UT}$ and M is calculated from radiosonde data at $00 \mathrm{UT}$. With all the available data of $\mathrm{Cn} 2$ a function of $\mathrm{L}_{0}$ with the height is retrieved as shown in Figure 1.

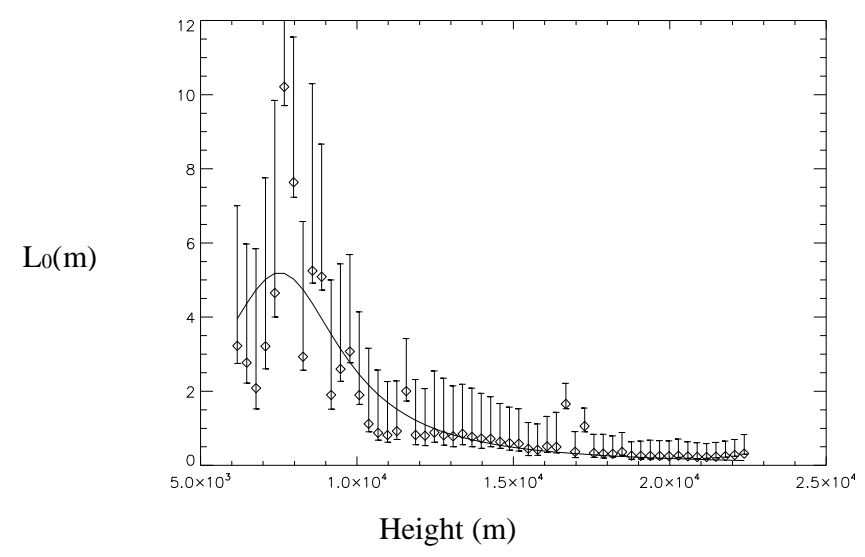

Figure 1. Outer Scale as a function of height at Teide Observatory obtained from Coulman's model

The relationship obtained for the Teide Observatory between $\mathrm{L}_{0}$ and $\mathrm{z}$ can be fitted as:

$$
L_{0}=\frac{5.2}{1+\left(\frac{z-7526}{2404}\right)^{2}}
$$

which is a similar to the expression given by Coulman.

Once the outer scale is known, day $\mathrm{Cn}^{2}$ profiles can be obtained using equation (1). As a first approach this model is valid although the main drawback is that $\mathrm{L}_{0}$ is assumed to be stationary during the day and depends on the height without considering atmospheric conditions.

\subsection{Dewan's Model}

Dewan et al. [5] proposed a model based on the relation between the outer scale and the statistical change of wind shear. A shear type instability leads to the formation of a turbulent layer. The outer scale is determined in a statistical manner from high resolution wind shear data.

A turbulent instability is defined by this condition:

$$
S_{c}^{2}>\frac{N^{2}}{2}
$$

where $\mathrm{N}$ is the buoyancy frequency and $\mathrm{S}$ is the wind shear defined as:

$$
S=\left[\left(\frac{d V_{x}}{d x}\right)^{2}+\left(\frac{d V_{y}}{d y}\right)^{2}\right]
$$

with Vx and Vy the wind velocity components. 
Two regimes are defined, the troposphere, with an S limit of 0.02, and stratosphere, with an S limit of 0.045 . Then all regions exceeding the limit, $S_{c}$ are presumed to be turbulent.

Dewan obtained a linear regression between the outer scale and wind shear:

$$
\begin{aligned}
& L_{0}^{4 / 3}=0,1^{4 / 3} * 10^{(1,64+42 * S)} \text { Troposphere } \\
& L_{0}^{4 / 3}=0,1^{4 / 3} * 10^{(0,5+50 * S)} \text { Stratosphere }
\end{aligned}
$$

The implementation of this model requires to know the tropopause limit for each turbulent profile. This limit is calculated following the exact definition used by the World Meteorological Organization:

"The boundary between the troposphere and the stratosphere where an abrupt change in lapse rate usually occurs. It is defined as the lowest level at which the lapse rate decreases to $2{ }^{\circ} \mathrm{C} / \mathrm{km}$ or less, provided that the average lapse rate between this level and all higher levels within $2 \mathrm{~km}$ does not exceed $2{ }^{\circ} \mathrm{C} / \mathrm{km} "$

Figure 2 shows the variation of the temperature with height where the tropopause is clearly identified.

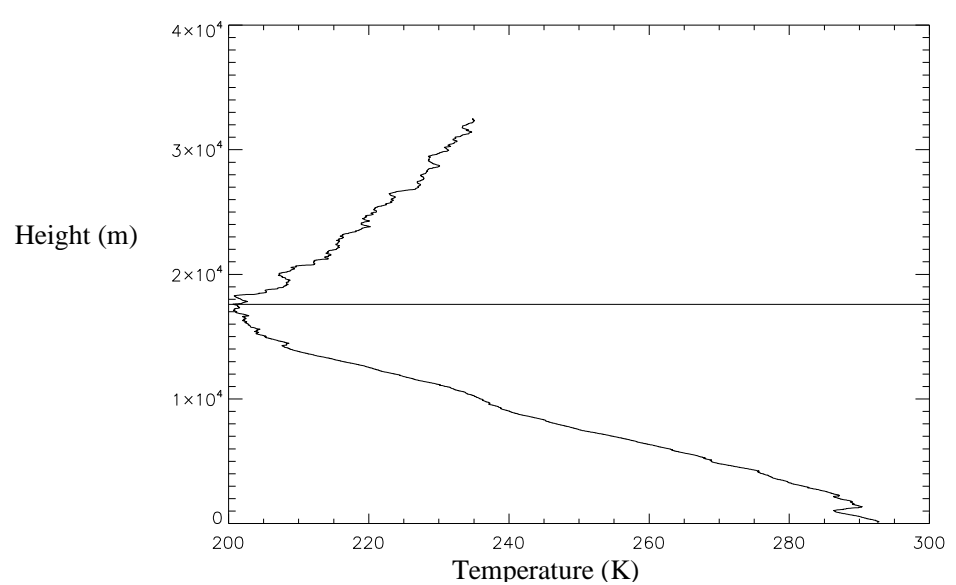

Figure 2. Temperature as a function of Height. The line represents the tropopause level

$\mathrm{Cn}^{2}$ is then derived from equation (1) setting $\gamma$ to 2.8 and $\mathrm{M}$ is calculated as:

$$
\boldsymbol{M}=-\left(\frac{78 \times 10^{-6} P}{T^{2}}\right)\left(\frac{\partial T}{\partial z}+\curlyvee\right)
$$

where $\Upsilon$ is the dry adiabatic lapse rate of $9.810^{3} \mathrm{~K} / \mathrm{m}$.

\subsection{Masciadri’s Model}

Masciadri et al. [7] present a modified version of model of Dewan. The outer scale is derived as done by Dewan, while $\mathrm{M}$ is computed:

$$
\boldsymbol{M}=-\left(\frac{78 \times 10^{-6} P}{\theta T}\right) \frac{\partial \theta}{\partial z}
$$

where $\theta$ is the potential temperature in $\mathrm{K}$. In this model $\gamma$ depends on the thermal and dynamic stability of the atmosphere, proportional to the inverse of the Prandtl number, and takes values from 0.78 in a stable layer to 2.2 in a very unstable layer. We took an intermediate value of 1.5. 


\subsection{Thorpe's Model}

Thorpe [6] proposed a model based on the measure of turbulence lengths from overturns in the gradient of water density in oceans and lakes. The same principle can be applied to the atmosphere [9]. The method is based on the comparison between an observed vertical profile of potential temperature and a reference profile corresponding to a minimum state of available potential energy. The reference profile is built by sorting in increasing order the observed potential temperature. The Thorpe signal is defined as $\left|Z_{\mathrm{obs}}-\mathrm{Z}_{\text {sort }}\right|$ being $\mathrm{Z}_{\mathrm{obs}}$ the height of the observed potential temperature and $Z_{\text {sort }}$ the height of the observed potential temperature in the sorted profile. In this study the Thorpe length, $\mathrm{L}_{\mathrm{T}}$, is taken as the absolute value of the Thorpe signal although in the literature is often used as the root mean square value of the Thorpe distance.

This method is just valid for the free atmosphere and regions statistically stable. In the boundary layer the overturning scale is of the order of 1/10 meters, while radiosondes have a resolution of 10 meters. Therefore this method is only valid for the free atmosphere where the overturning scale is larger than 10 meters.
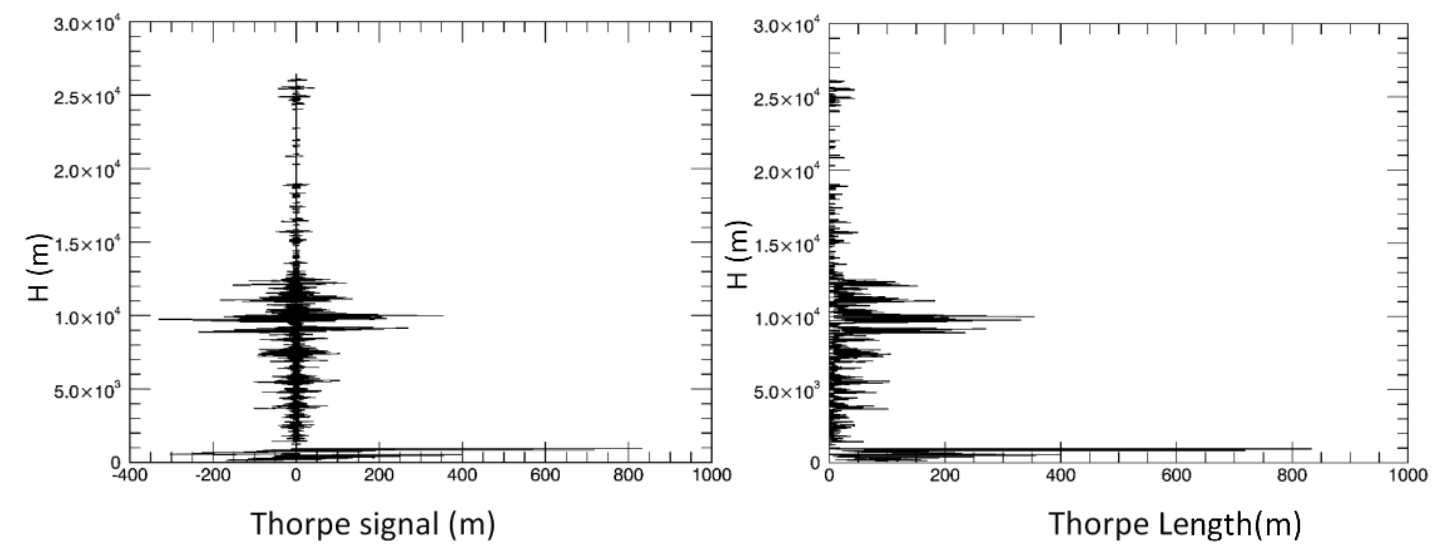

Figure 3.Left: Thorpe signal, Right: Thorpe length.

In Figure 3 we show the Thorpe signal and Thorpe length for a certain profile. Several gaps appear due to the lack of resolution of the radiosonde data under strong stratification. In order to fill those gaps we study the relation between $d \theta / d z$ and $L_{T}$. A power law regression is used to extrapolate $L_{T}$ values as shown in Figure 4. The gap-filled $L_{T}$ profile is shown in red in Figure 4.
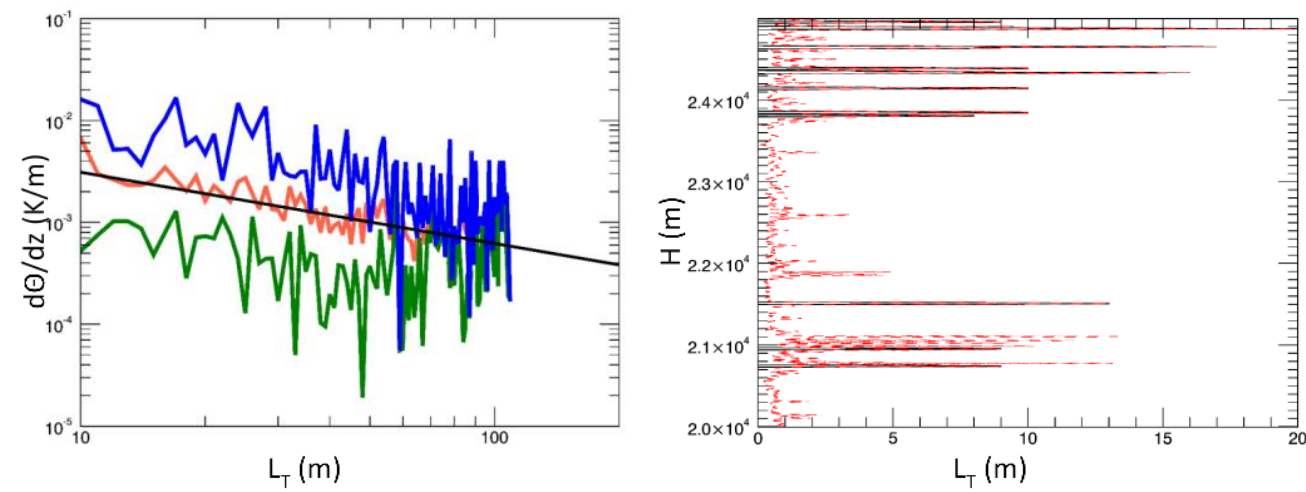

Figure 4. Left: Dependence of gradient of potential temperature with Thorpe length. Right: Gap-filled Thorpe length in red.

This model uses the same expression as Masciadri et al. [7] for M (equation 8) and the best value found for $\gamma$ is 1.2. 
Trinquet and Vernin [9] use an alternative approach to Tatarski [1]. They calculate $\mathrm{Cn}^{2}$ from the temperature structure constant $\mathrm{Ct}^{2}$. Both quantities are related by the Gladstone equation [7]:

$$
C_{n}^{2}=\left(\frac{80 \times 10^{-6} P}{T^{2}}\right)^{2} C_{T}^{2}
$$

A statistical study shows that $\mathrm{Ct}^{2}$ is proportional to the vertical wind shear $\mathrm{S}(\mathrm{z})$ and the buoyancy frequency $\chi(\mathrm{z})$ following [9]:

$$
C_{T}^{2}=\phi(z) \chi(z) S(z)^{1 / 2}
$$

where $\phi(z)$ is a parameter that has been measured experimentally in La Palma [9].

The main difference with the models based on Tatarski's equation is that $\mathrm{Cn}^{2}$ is proportional to $\chi$ instead of $\chi^{2}$.

In the next section we compare the data obtained with SCIDAR instruments with the models described above.

\section{MODEL COMPARISON}

The aim of this work is to define the best model for deriving $\mathrm{Cn}^{2}$ profiles from radiosonde data. We use the SCIDAR data as a reference for comparison. In this section we compare the night time data from SCIDAR averaged from 00 to 01 UT with radiosonde data obtained at $00 \mathrm{UT}$.

It has to be noticed that the balloon is launched at Güimar station approximately from sea level, while the SCIDAR instrument is placed at the observatory level at 2362 meters. Therefore we can only compare the free atmosphere values, while the radiosonde is not sensitive to the boundary layer.

Due to the variability of the data we compare the interquartile range (IQR defined as the difference between the first quartile and third quartile of a set of data) for each set of data.

\subsection{Comparison of HR and LR data}

As described in section 1, we have access to radiosonde data with different resolution. We have applied Dewan's model to both sets of data. In Figure 5 we represent the interquartile range for night time data with LR in blue and night time data with HR in red. We expected to have the same curve but there is a disagreement for low altitudes while there is a better agreement for high altitudes. This mismatch is due to the difference on the calculation of the tropopause level and to the fact that $\mathrm{L}_{0}$ in Dewan's model is fitted to LR data. Therefore to ensure the validity of the model for comparison with other methods we will take LR data. 


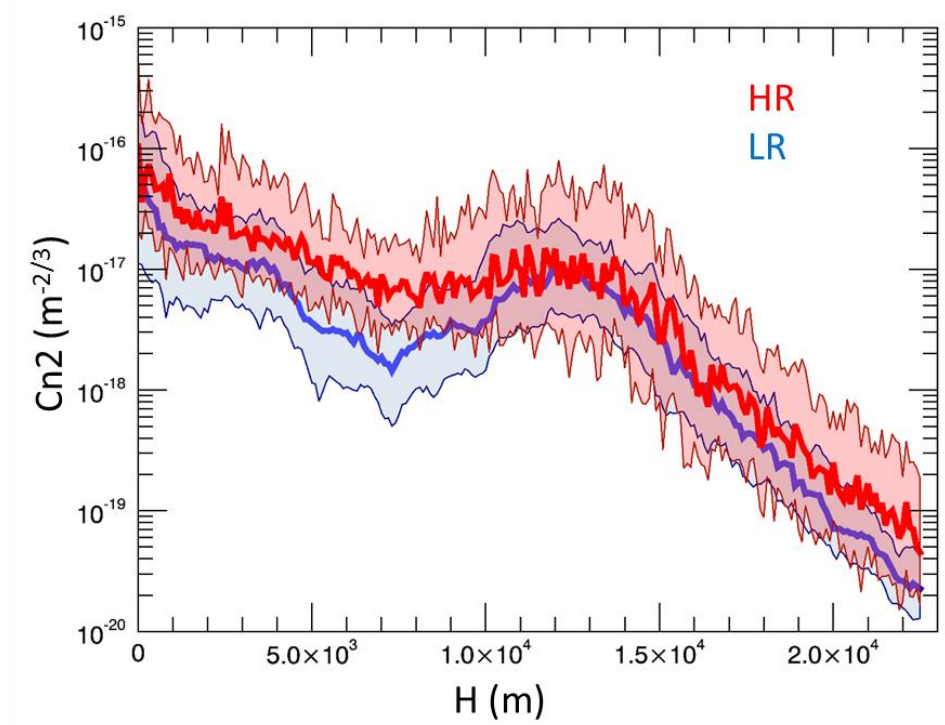

Figure 5. $\mathrm{Cn}^{2}$ profile obtained using Dewan's model for night time radiosonde data. Red: high resolution data. Blue: low resolution data

\subsection{Comparison Night and Day time data}

Figure 6 shows a single profile in the same day, for day and night, for the free atmosphere using Dewan's model. The profiles are very similar. Indeed for the whole set of data, the median value for day and night is almost the same.

The important conclusion from this analysis is that the free atmosphere can be considered stationary during the day. Therefore we can use as day time free atmosphere profiles those obtained from SCIDAR or other optical instrumental at the observatory during the night. We can also use the free atmosphere profiles obtained at 12UT with radiosonde model.
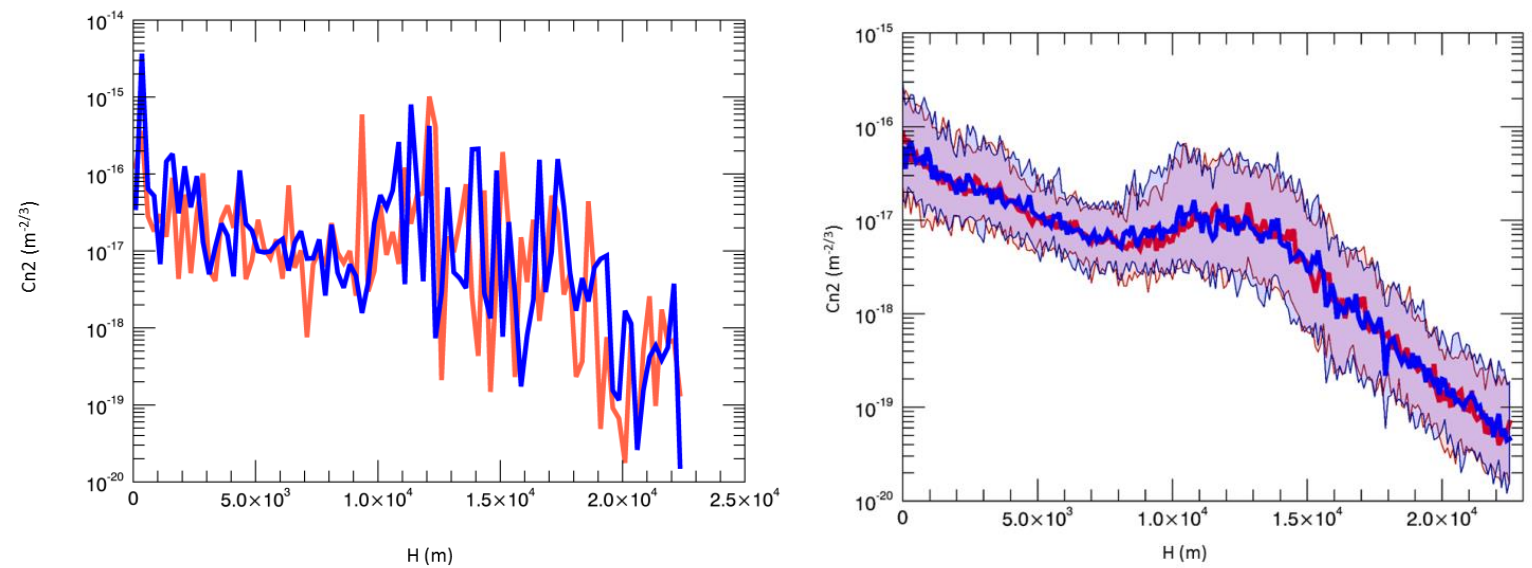

Figure 6.Comparison of $\mathrm{Cn}^{2}$ profiles in the free atmosphere using Dewan's model for day (red) and night(blue). Left: individual profile. Right: IQR 


\subsection{Model comparison with SCIDAR}

In Figure 7 we represent the interquartile for SCIDAR data (red) compared with the radiosonde models (blue) described in section 3 .

From this analysis we derive that:

- Dewan's model is able to retrieve the turbulent layers, although there is an offset between both sets of data. The offset may be reduced by adjusting the constant $\Phi$.

- Masciadri's model is the one that best fits the SCIDAR data.

- Thorpe's method is not sensitive to high turbulent layers. This is due to the lack of resolution of the data and the error introduced to determine the inversions in the potential temperature profile. This model may be improved by de-noising the profiles. There are several methods to de-noise the profiles:

1. The simplest one is to apply a moving average filter to the potential temperature.

2. The cumulative sum of the potential temperature can be used to identify the inversion layer? and calculate the rms of the Thorpe displacement.

3. Wilson et al. [11] propose a deep analysis of the trend noise ratio to distinguish between the real atmospheric inversions and the overturns introduced by instrument noise.

- Trinquet's model agrees with SCIDAR data for low altitudes but diverges for high turbulent layers.
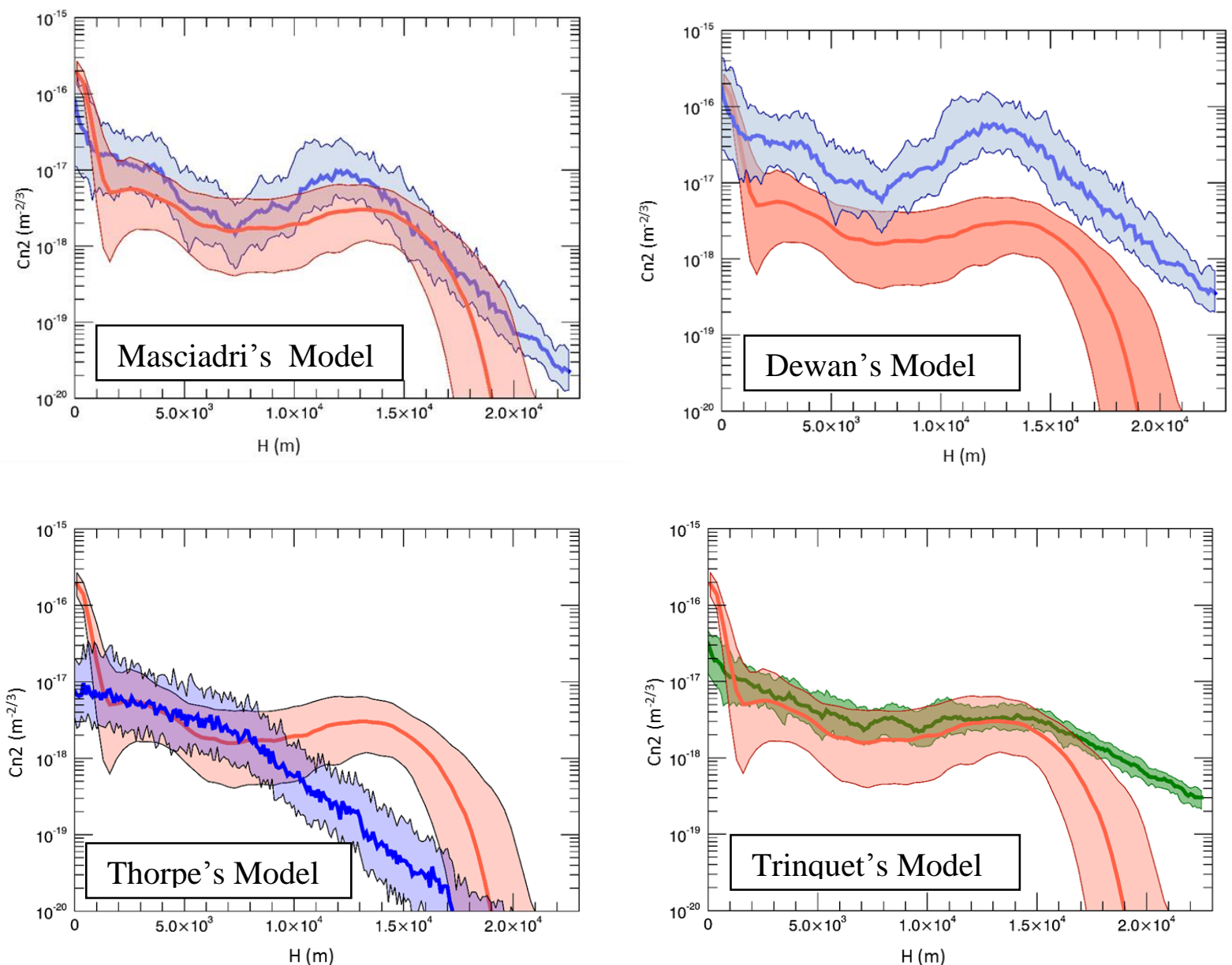

Figure 7.Comparison of $\mathrm{Cn}^{2}$ profiles using radiosonde models (blue and green for Trinquet's Model) and SCIDAR data (red). 


\section{MODELING DAY TIME $\mathrm{CN}^{2}$ PROFILE}

In this section we describe how to build a synthetic day time profile for the MCAO simulations.

As mentioned in section 1, SHABAR data provide $\mathrm{Cn}^{2}$ profiles during day time [2]. As an example, Figure 8 shows the evolution of $\mathrm{Cn}^{2}$ during one day. As expected, turbulence is stronger at noon. It can be noticed that above around $2000 \mathrm{~m}$ there is no structure on $\mathrm{Cn}^{2}$. The instrument provides reliable $\mathrm{Cn}^{2}$ information up to $2000 \mathrm{~m}$ which is the limit of the boundary layer.

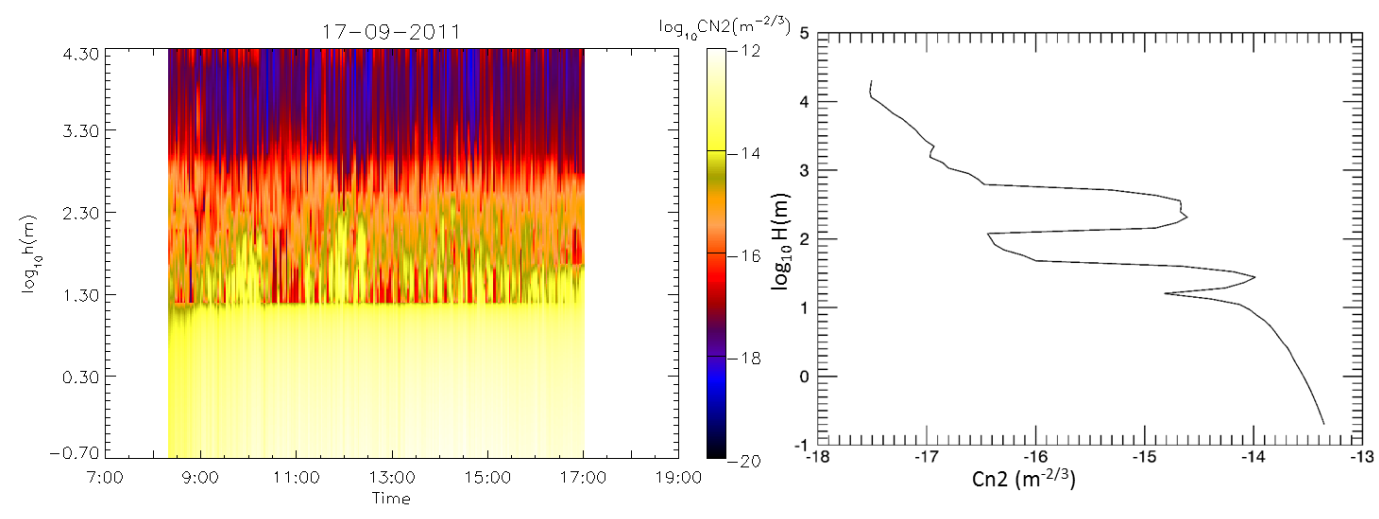

Figure 8. Left: Temporal evolution of $\mathrm{Cn}^{2}$ profiles during a particular day. Right: individual $\mathrm{Cn}^{2}$ profile.

The synthetic profile is built by a combination of SHABAR data for the boundary layer and radiosonde data obtained at noon for the free atmosphere following the equation:

$$
C_{n_{-} T O T A L}^{2}(0-H)=C_{n_{-} S H A B A R}^{2}(0-2 K m)+C_{n_{\_} \text {Model }}^{2}(2 K m-H) \frac{\int_{2 K m}^{H} C_{n_{-S H A B A R}}^{2}(h) d h}{\int_{2 K m}^{H} C_{n_{-M o d e l}}^{2}(h) d h}
$$

Figure 9 shows an example of the resulting profile using Masciadri's model for the free atmosphere. We have created a data base of day-time vertical $\mathrm{Cn}^{2}$ profiles which will be used for the simulation of MCAO.

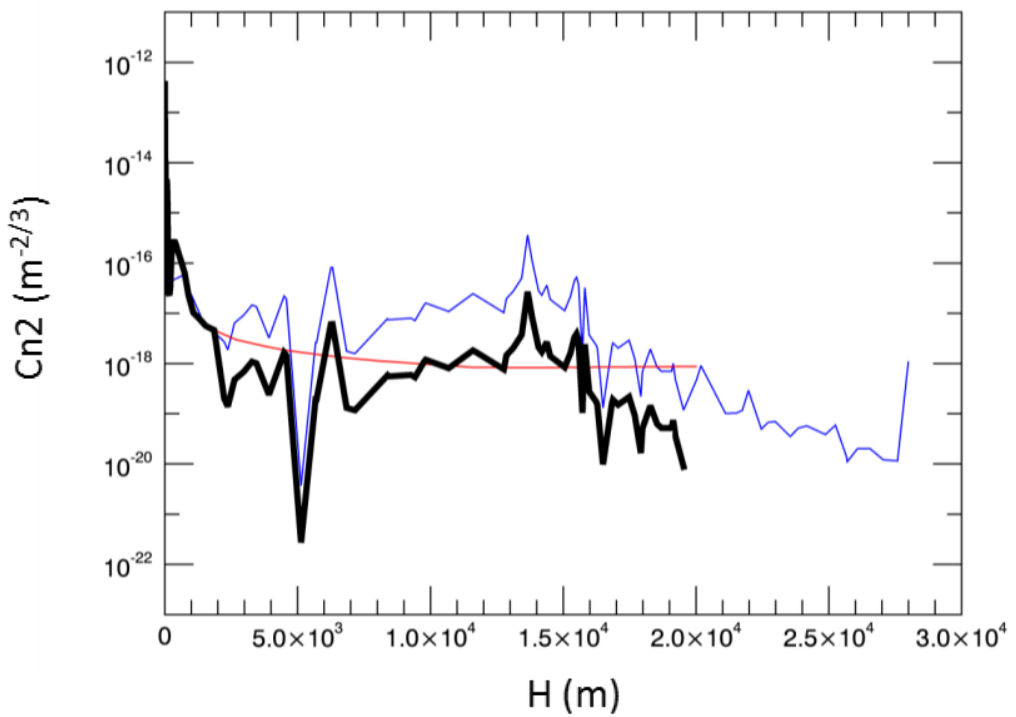

Figure 9. Vertical $\mathrm{Cn}^{2}$ profiles for SHABAR (red), radiosonde (blue), combination SHABAR-Radiosonde (black) 


\section{CONCLUSION}

We have performed a study of different models to retrieve $\mathrm{Cn}^{2}$ vertical profiles from radiosonde data. Four models have been studied and compared with data obtained from the SCIDAR instrument. The model that best fits SCIDAR data is the one proposed by Masciadri et al. [7]. We have confirmed that the turbulence in the free atmosphere is almost stationary (i.e., it does not depend on the day-night cycle). This is the most important result derived from this study, since it allows to build vertical turbulence profiles using day time ground layer profiles obtained with the SHABAR instrument combined with free atmosphere profiles obtained from radiosonde models. This stratification allows us to perform simulations of MCAO at any time of the day using real turbulence profiles.

\section{REFERENCES}

[1] V. I. Tatarski, "Wave Propagation in a Turbulent Medium”, McGraw-Hill, New York, 1961.

[2] García-Lorenzo, B.; Fuensalida, J. J. "Statistical structure of the atmospheric optical turbulence at Teide Observatory from recalibrated generalized SCIDAR data" MNRAS,410,2011.

[3] J. Marco de la Rosa; L. Montoya; M. Collados; I. Montilla; N. Vega Reyes "Daytime turbulence profiling for EST and its impact in the solar MCAO system design” Proc. SPIE 9909, Adaptive Optics Systems V, 99096X,2016.

[4] Coulman C,Vernin J, Coqueugniot Y, Caccia J "Outer scale of turbulence appropriate to modelling refractive-index structure profiles". Appl Opt 27,1998.

[5] Dewan, E. M., R. E. Good, B. Beland, and J. Brow “ A Model for (Optical Turbulence Profiles using Radiosonde Data” Phillips Laboratory Technical Report, PL-TR-93-2043. ADA 279399,1993.

[6] Thorpe, “Turbulence and Mixing in a Scottish Loch”. Philosophical Transactions of The Royal Society B Biological Sciences 286,1977.

[7] Masciadri, E.; Lascaux, F.; Turchi, A.; Fini, L.“Optical turbulence forecast: ready for an operational application”. MNRAS 466, 2017.

[8] Sukanta Basu, "A simple approach for estimating the refractive index structure parameter (Cn2) profile in the atmosphere". Opt. Lett. 40, 2015.

[9] Hervé Trinquet,Jean Vernin “A statistical model to forecast the profile of the index structure constant Cn2", Environ Fluid Mech 3972007.

[10] Giordano, C.; Vernin, J.; Trinquet, H.; Muñoz-Tuñón, C. "Weather Research and Forecasting prevision model as a tool to search for the best sites for astronomy: application to La Palma, Canary Islands". MNRAS 440,2014.

[11] R. Wilson, F. Dalaudier, H. Luce "Can one detect small-scale turbulence from standard meteorological radiosondes?” Atmos. Meas. Tech., 4, 2011. 